\title{
Meningioma with Metastatic Malignant Melanoma: An Unusual Coincidence
}

\author{
1 Department of Neurosurgery, BYL Nair Hospital and TN Medical \\ College, Mumbai, Maharashtra, India \\ Indian J Neurosurg 2017;6:213-215.
}

H. V. Savant ${ }^{1}$ Anand Doshi ${ }^{1}$ Zafar H. Sheikh ${ }^{1} \quad$ Vijay Mahajan $^{1}$

\begin{abstract}
Address for correspondence Zafar H. Sheikh, MS, MCh, Department of Neurosurgery, BYL Nair Hospital and TN Medical College, Dr Anandrao Nair Marg, Mumbai, Maharashtra 400008, India (e-mail: zafar18@gmail.com).
\end{abstract}

\begin{abstract}
Simultaneous occurrence of meningioma and brain metastasis as two different histopathologically-proven lesions has been often reported in literature, however, to the best of our knowledge, contiguous occurrence of meningioma and malignant melanoma has not been reported previously in literature. A 50-year-old woman presented with features of raised intracranial pressure and left upper motor neuron signs. Imaging findings were suggestive of right frontoparietal and right parietal extra-axial space occupying extra-axial lesions. Patient underwent right frontotemporoparietal craniotomy with excision of both tumors under general anesthesia. Histopathological analysis was suggestive of transitional meningioma

Keywords

- dual tumors

- meningioma

- metastatic melanocytoma

(World Health Organization [WHO] grade I) and malignant melanoma (WHO grade IV). Simultaneous presence of two tumors in the brain might be of the same or different histopathology. In such dual tumors, metastasis cases are varied and one source of it may be melanoma of the skin. Hence, dermatological examination can reveal the primary source of tumor in select cases.
\end{abstract}

\section{Introduction}

Meningiomas are the tumors which are found in cases of multiple intracranial tumors of different histology. This is attributed to frequent occurrence of intracranial meningiomas and their long clinical evolution before diagnosis. Thus, they have an increased probability of simultaneously harboring another primary or secondary intracranial tumors. Central nervous system (CNS) melanotic tumors include the rare primary melanomas and the more common secondary melanomatous deposits. ${ }^{1}$ Melanoma ranks fourth in the incidence of brain metastases. ${ }^{2}$ We present a case which involves two separate right convexity tumors. Histopathology of the excised specimens revealed two histologically different tumors: a transitional meningioma and second metastatic melanoma. Simultaneous occurrence of meningioma and brain metastasis as two different histological proven lesions has been reported in various literature, ${ }^{3,4}$ however, to the best of our knowledge, contiguous occurrence of meningioma and malignant melanoma has not been reported previously in literature. We would like to document this as the first case.

\section{Case Report}

A 50-year-old woman was admitted with complaints of headache since 2 months, forgetfulness, and walking instability since 10 to 15 days which was associated with vomiting. Eventually her alertness decreased with slowing of activities. On examination, she was conscious, obeying commands but disoriented in time and place, recognized relatives, and preferred to sleep. She had left-sided upper motor neuron signs, hypertonia, and brisk reflexes with plantar dorsiflexors associated with upper limb drift due to weakness and left upper motor facial paresis. Her hematological and biochemical investigations were within normal limits. Computed tomography (CT) of the brain with contrast suggestive of right frontoparietal and right parietal extra-axial space occupying lesion with homogenous received

June 24, 2015

accepted

July 25, 2016

published online

May 9, 2017
DOI https://doi.org/

$10.1055 / \mathrm{s}-0036-1593604$. ISSN 2277-954X. (c) 2017 Neurological Surgeons' Society of India
License terms

(요 (1) $\Theta$ 
contrast enhancement with central nonenhancing area and perilesional edema causing mass effect ( - Fig. 1A). Magnetic resonance imaging (MRI) of the brain showed two extraaxial lesions which were isointense on T1 (-Fig. 1B), mildly hyperintense on T2 (-Fig. 1C), and exhibited heterogeneous enhancement (-Fig. 1D).

Patient underwent right frontotemporoparietal craniotomy with excision of both tumors under general anesthesia. The right parietal tumor was found attached to dura necessitating circumferential excision of $1 \mathrm{~cm}$ margin of the latter. The tumor was sent for frozen section and reported to be meningioma. Right parietal tumor was approached via arachnoid plane and excised totally. Right frontoparietal tumor was bluish-black in color, no obvious arachnoid plane was seen, and it was highly vascular and was bleeding on holding the tumor by forceps. Frozen section reported as melanocytic tumor. Piece meal excision of tumor was done. Brain was lax and pulsating.

Postoperatively, patient showed significant improvement in her neurological status. Histopathological analysis was suggestive of transitional meningioma (WHO grade I) (-Fig. 2A) and malignant melanoma (WHO grade IV) (-Fig. 2B). A retrospective clinical evaluation showed primary skin lesion at the nape of neck ( - Fig. 3) which was confirmed with biopsy ( - Fig. 2C). CT scan of the abdomen revealed liver metastasis. CA 19.9 and CA 125 were found raised. Eventually, the patient died 1 month postoperatively

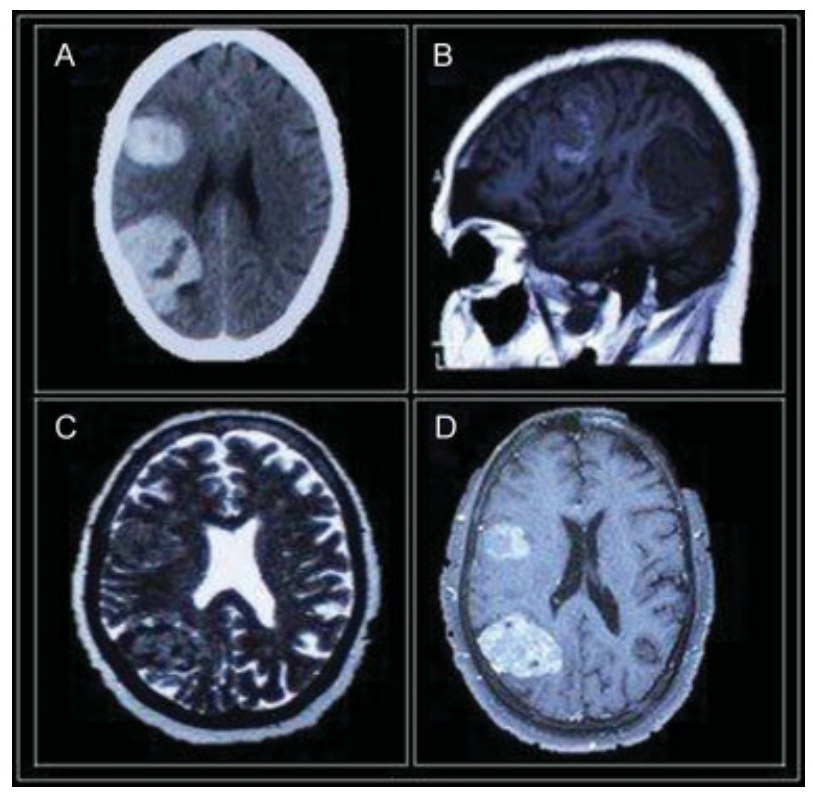

Fig. 1 (A) Computed tomography of the brain with contrast study reveals right frontoparietal and right parietal extra-axial space occupying lesions with homogenous contrast enhancement and central nonenhancing areas causing mass effect. (B) T1-weighted sagittal section of magnetic resonance imaging of the brain reveals right frontoparietal lesion is iso- to heterogeneously hyperintense while right parietal lesion is hypointense. (C) T2-weighted axial section of magnetic resonance imaging of the brain reveals both lesion which are mildly hyper intense. (D) Gadolinium contrast magnetic resonance imaging of the brain reveals both lesions which are heterogeneously enhancing

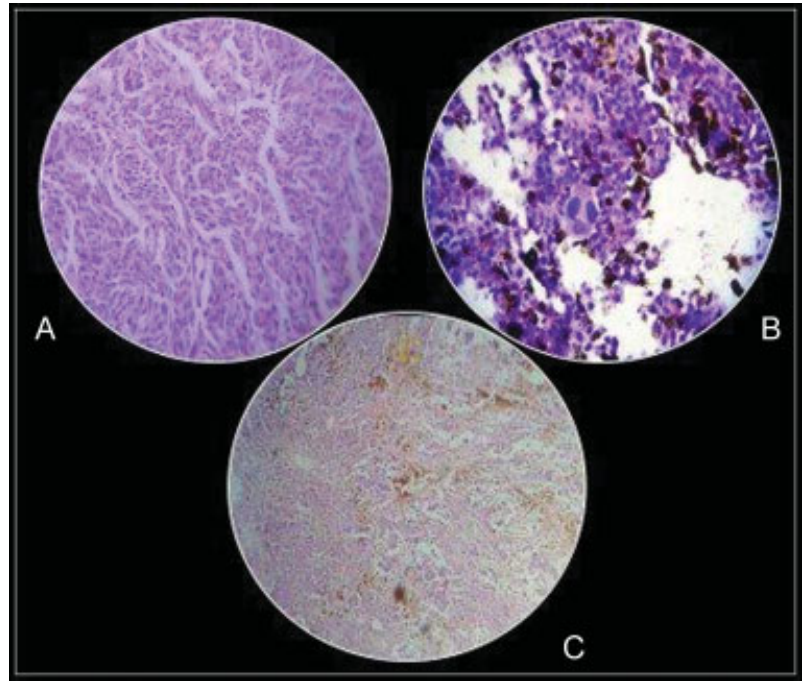

Fig. 2 Slide (A) hematoxylin and eosin histological sections seen at magnification $\times 40$. Microscopic examination showed a meningothelial tumor of transitional morphology. The nuclei exhibited pleomorphism and hyperchromasia. MIB-1 index approximately 23\%. Slide (B) hematoxylin and eosin histological sections seen at magnification $\times 40$. Microscopic examination showed a high-grade malignant tumor admixed with melanincontaining tumor cells. Mitotic activity noted. IHC: positive for S-100 and HMB-45; MIB-1 index approximately 40 to $50 \%$. Slide (C) skin biopsy hematoxylin and eosin histological sections seen at magnification $\times 10$. Microscopic examination showed a tumor infiltrating into the surrounding connective tissue. Tumor cell with melanin pigments.

because of acute respiratory failure due to pulmonary edema, hypoalbuminemia, and electrolyte disturbance.

\section{Discussion}

Primary brain tumors with different histological types is only $0.3 \%$ of all brain tumors.

The spatial relationship between a meningioma and a single brain metastasis may include three different possibilities: the metastasis may be harbored within the meningioma, in the surrounding brain parenchyma, and elsewhere in the brain. The female:male ratio is approximately $2: 1$, and is rare in prepubertal age group. ${ }^{5}$ Atypical and malignant meningioma comprise a small fraction with slight male predominance. ${ }^{5}$

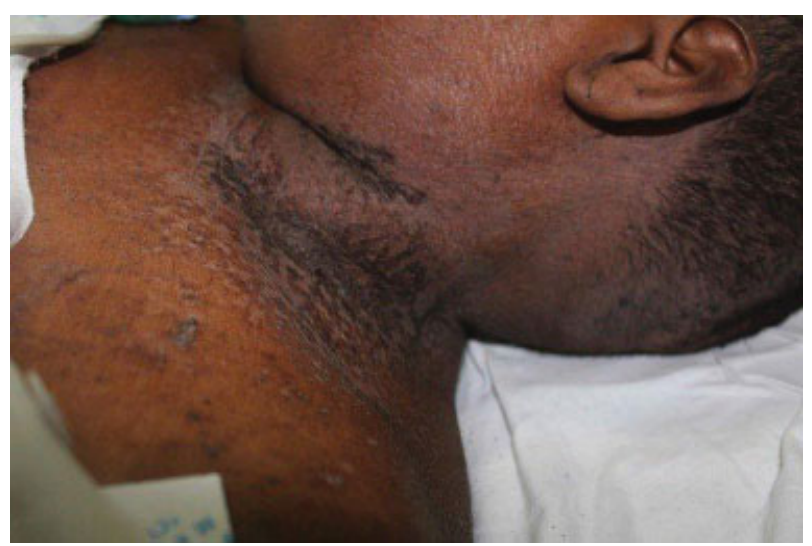

Fig. 3 Blackish pigmented patch seen over the left lateral aspect of neck. 
CNS melanotic tumors include the rare primary melanomas and the more common secondary melanomatous deposits. ${ }^{1}$ Primary CNS melanomas are rare with estimated incidence of 0.9 per 10 million. $^{1}$ Peak incidence of these tumors is in the fourth and fifth decades, with more of male predominance. ${ }^{6}$ Melanoma ranks fourth in the incidence of brain metastases, behind lung, breast, and unknown primary cancers, ${ }^{2}$ representing $9 \%$ of all CNS metastasis. ${ }^{7}$ As such brain has been referred to as a sanctuary site for melanoma metastasis.

Epidemiologic studies suggest that metastasis of the brain is inversely correlated with metastasis to the liver suggesting these processes may be mediated in different ways. ${ }^{8}$ Liver metastases is usually the late stage of disease with an average lifespan of only 2 to 4 months. ${ }^{8}$

Cutaneous melanoma metastasizing to brain has very poor prognosis with an overall median survival of only 6 to 10 months and a 5-year survival of less than $10 \%{ }^{9}$ An analysis of 355 patients with melanoma brain metastases diagnosed from 1991 to 2001 and treated at the Memorial Sloan Kettering Cancer Center (MSKCC) reported a median overall survival of 5.2 months. $^{10}$

Usual presentation is intracranial hemorrhages in approximately $50 \%$ of patients, 8 most common site of lesions seen are in frontal and parietal lobes. ${ }^{8}$ Metastatic frequency to both hemispheres are equal. ${ }^{8}$ Sometimes there may be brain metastasis with unknown primary, its incidence is reported between 2 to $10 \% .{ }^{11}$ It may be secondary due to de novo melanoma within lymph node, respiratory or gastrointestinal tract, vagina, or spontaneous regression of primary lesion. ${ }^{11}$ Histologically, tumors of melanocytic origin range from benign melanocytomas to malignant melanomas. ${ }^{7}$

There are currently no FDA (U.S. Food and Drug Administration)-approved therapies which significantly improve overall survival for patients with late-stage disease. ${ }^{12}$ Several clinical trials have reported low response rates and poor survival for patients with melanoma brain metastases who are treated with systemic therapies. ${ }^{13,14}$

Simultaneous presence of two tumors in the brain might be of the same or different histopathology. In such metastasis, cases are varied and one source of it may be melanoma of the skin. Hence, dermatological examination is also warranted in such cases.

\section{References}

1 Atkins MB, Sosman JA, Agarwala S, et al. Temozolomide, thalidomide, and whole brain radiation therapy for patients with brain metastasis from metastatic melanoma: a phase II Cytokine Working Group study. Cancer 2008;113(8): 2139-2145

2 Schlagenhauff B, Stroebel W, Ellwanger U, et al. Metastatic melanoma of unknown primary origin shows prognostic similarities to regional metastatic melanoma: recommendations for initial staging examinations. Cancer 1997;80(1):60-65

3 Balch CM, Houghton AN, Sober AJ, Soong SJ, Atkins MB, Thompson JF. Cutaneous Melanoma. 4th ed. St. Louis, MO: Quality Medical Publishing; 2003

4 Raizer JJ, Hwu WJ, Panageas KS, et al. Brain and leptomeningeal metastases from cutaneous melanoma: survival outcomes based on clinical features. Neuro-oncol 2008;10(2):199-207

5 Maiuri F, Cappabianca P, Iaconetta G, Esposito F, Messina A. Simultaneous presentation of meningiomas with other intracranial tumours. Br J Neurosurg 2005;19(4):368-375

6 Tokunaga T, Shigemori M, Hirohata M, Sugita Y, Miyagi J, Kuramoto S. Multiple primary brain tumors of different histological types-report of two cases. Neurol Med Chir (Tokyo) 1991;31(3):141-145

7 Burger P, Scheihauer BW, Vogel FC. Intracranial meninges, In: Surgical pathology of nervous system and its coverings. 4th ed. Philadelphia, Churchill Livingston: 49-712002

8 Seckin H, Yigitkanli K, Ilhan O, Han U, Bavbek M. Breast carcinoma metastasis and meningioma. A case report. Surg Neurol 2006;66(3):324-327, discussion 327

9 Darnell RB, Posner JB. Paraneoplastic syndromes affecting the nervous system. Semin Oncol 2006;33(3):270-298

10 Ko JM, Fisher DE; KoJM. A new era: melanoma genetics and therapeutics. J Pathol 2011;223(2):241-250

11 Agarwala SS, Kirkwood JM, Gore M, et al. Temozolomide for the treatment of brain metastases associated with metastatic melanoma: a phase II study. J Clin Oncol 2004;22(11): 2101-2107

12 Korn EL, Liu PY, Lee SJ, et al. Meta-analysis of phase II cooperative group trials in metastatic stage IV melanoma to determine progression-free and overall survival benchmarks for future phase II trials. J Clin Oncol 2008;26(4):527-534

13 Schuchter LM, Haluska F, Fraker D, Elenitsas R. Skin: malignant melanoma. In: Abeloff MD, ed. Clinical Oncology. Philadelphia: Churchill Livingstone; 1326-72000

14 Wen PY, Black PM, Loeffler JS. "Metastatic brain cancer," in Cancer: Principles and Practice of Oncology. In: DeVita VT, Hellman S, Rosenberg SA, eds., 6th edition. Philadelphia, PA: Lippincott, Williams \& Wilkins; 2001:2655-2670 